OPEN ACCESS

Edited by:

Vishwanath Venketaraman, Western University of Health Sciences, USA

Reviewed by: Yongqun $\mathrm{He}$, Virginia Tech, USA Birgit Strobl,

University of Veterinary Medicine Vienna, Austria

${ }^{*}$ Correspondence: António G. Castro, Life and Health Sciences Research Institute (ICVS), School of Health Sciences, University of Minho, Campus de Gualtar, Braga 4710-057, Portugal acastro@ecsaude.uminho.pt

tPresent address: Andrea Cruz and Margarida Saraiva, Institute for Molecular and Cell Biology (IBMC), University of Porto, Porto, Portugal

Specialty section: This article was submitted to

Microbial Immunology, a section of the journal

Frontiers in Immunology

Received: 16 June 2015 Accepted: 14 September 2015 Published: 30 September 2015

Citation:

Cruz A, Ludovico P, Torrado E, Gama JB, Sousa J, Gaifem J, Appelberg $R$, Rodrigues $F$, Cooper AM, Pedrosa J, Saraiva M and Castro AG (2015) IL-17A

promotes intracellular growth of

Mycobacterium by inhibiting apoptosis of infected macrophages.

Front. Immunol. 6:498. doi: 10.3389/fimmu.2015.00498

\section{IL-17A promotes intracellular growth of Mycobacterium by inhibiting apoptosis of infected macrophages}

\author{
Andrea Cruz ${ }^{1,2 \dagger}$, Paula Ludovico ${ }^{1,2}$, Egidio Torrado ${ }^{1,2,3}$, José Bernardo Gama ${ }^{1,2}$, \\ Jeremy Sousa ${ }^{1,2}$, Joana Gaifem ${ }^{1,2}$, Rui Appelberg ${ }^{4}$, Fernando Rodrigues ${ }^{1,2}$, \\ Andrea M. Cooper ${ }^{3}$, Jorge Pedrosa ${ }^{1,2}$, Margarida Saraiva $^{1,2+}$ and António G. Castro ${ }^{1,2 *}$ \\ ${ }^{1}$ Life and Health Sciences Research Institute (ICVS), School of Health Sciences, University of Minho, Braga, Portugal, \\ ${ }^{2}$ ICVS/3B's - PT Government Associate Laboratory, University of Minho, Braga, Portugal, ${ }^{3}$ Trudeau Institute, Saranac Lake, \\ NY, USA, ${ }^{4}$ Department of Immunophysiology, University of Porto, Porto, Portugal
}

The fate of infected macrophages is a critical aspect of immunity to mycobacteria. By depriving the pathogen of its intracellular niche, apoptotic death of the infected macrophage has been shown to be an important mechanism to control bacterial growth. Here, we show that IL-17 inhibits apoptosis of Mycobacterium bovis BCG- or Mycobacterium tuberculosis-infected macrophages thus hampering their ability to control bacterial growth. Mechanistically, we show that IL-17 inhibits p53, and impacts on the intrinsic apoptotic pathway, by increasing the Bcl2 and decreasing Bax expression, decreasing cytochrome $c$ release from the mitochondria, and inhibiting caspase- 3 activation. The same effect of IL-17 was observed in infected macrophages upon blockade of p53 nuclear translocation. These results reveal a previously unappreciated role for the IL-17/ p53 axis in the regulation of mycobacteria-induced apoptosis and can have important implications in a broad spectrum of diseases where apoptosis of the infected cell is an important host defense mechanism.

Keywords: IL-17, p53, apoptosis, mycobacteria, macrophages

\section{Introduction}

Mycobacterium tuberculosis is an intracellular pathogen responsible for the death of $\sim 1.5$ million people every year (1). Macrophages are the first cells to be infected by $M$. tuberculosis and provide the bases for a sequence of microbicidal actions which contain the infection (2). A growing body of evidence points to the ability to manipulate apoptosis, a form of programed cell death, as an important determinant of mycobacterial pathogenesis, although conflicting results support either inhibition or activation of apoptosis as a virulence mechanism $(3,4)$. Indeed, several studies suggest that the induction of apoptosis is unique to avirulent strains of mycobacteria, acting as a mechanism to prevent the spread of infection, by allowing antigen cross-presentation in favor of the host (5-8). In contrast, other authors argue that only virulent strains of $M$. tuberculosis induce apoptosis of infected cells $(3,9,10)$. These apparent discrepancies may be due to experimental conditions, with in vivo data suggesting that both apoptosis and necrosis of infected cells occur, depending on the anatomical location and timing of infection (3).

Among the factors dictating the fate of mycobacterially infected cells is the cytokine composition of the extracellular milieu. Specifically, tumor necrosis factor (TNF)- $\alpha$ induces while interleukin (IL)-10 
inhibits apoptosis of the mycobacterially infected macrophages (11, 12). Although M. tuberculosis-infected macrophages are exposed to many other cytokines produced during the immune response, the impact of these cytokines on apoptosis of infected cells remains unclear. In this context, IL-17 is interesting, as this cytokine produced by $\mathrm{T}$ helper (Th)17 cells during chronic viral infection leads to the upregulation of the pro-survival proteins $\mathrm{Bcl}-\mathrm{xl}$ and $\mathrm{Bcl} 2$ resulting in reduced apoptosis of infected cells; this, in turn, contributes to viral persistence and pathogenesis (13). IL-17 was also shown to promote survival of airway macrophages upon allergen-induced airway inflammation (14) and of fibroblast-like synoviocytes during chronic inflammation, through signal-transducer and activator of transcription protein (STAT)-3 activation (15). Moreover, IL-17 and Th17 cells have been linked to cancer, although both pro- and anti-tumorigenic activities have been ascribed to this cytokine (16-18).

The evidence supporting a role for IL-17 in modulating the apoptotic pathway, together with the early production of IL-17 following $M$. tuberculosis infection (19), prompted us to investigate the role of IL-17 in the modulation of apoptosis in mycobacterially infected macrophages. Using mouse primary bone marrow-derived macrophages (BMDM) infected with M. tuberculosis or M. bovis BCG, we show that in the presence of IL-17 macrophages are more permissive to Mycobacterium growth consistent with a reduced apoptotic death of the infected macrophages. Mechanistically, we show that IL-17 interferes with the master regulator of apoptosis p53, alters the transcriptional profile of $\mathrm{Bax}$ and $\mathrm{Bcl} 2$ and inhibits cytochrome $c$ release.

\section{Materials and Methods}

\section{Animals}

Eight- to 12-week-old female C57BL/6 mice obtained from Charles River (Barcelona, Spain) were used. All experimental protocols involving mice were performed according to the European Union Directive $86 / 609 /$ EEC, and previously approved by the national authority Direção Geral de Alimentação e Veterinária.

\section{Reagents and Bacteria}

Recombinant mouse IL-17A (R\&D Systems) and the specific p53 chemical inhibitor cyclic pifithrin-alpha (PFT- $\alpha$; Sigma) were used at $100 \mathrm{ng} / \mathrm{mL}$ and $30 \mu \mathrm{M}$, respectively, based on a dose response curve (data not shown). M. bovis BCG Pasteur or $M$. tuberculosis H37Rv (a kind gift of Pere-Juan Cardona, Barcelona) stocks were prepared as previously described (20).

\section{Culture of Mouse BMDM}

Bone marrow-derived macrophages were generated and infected at a multiplicity of infection (MOI) of two bacteria per macrophage as described $(20,21)$. Cells were treated as described in the figure legends. To determine the number of viable bacteria, macrophage cultures were lysed with $0.1 \%$ saponin and the bacterial suspensions were serially diluted and plated onto $7 \mathrm{H} 11$ agar medium. Bacterial colony formation was counted after 3 weeks of incubation at $37^{\circ} \mathrm{C}$. To determine the viability of BMDM in culture, the medium was aspirated and the adherent cells incubated for $10 \mathrm{~min}$ with $2.5 \mathrm{mg} / \mathrm{mL}$ of protease type XXV (Sigma) to digest any dead cells. Next, cetrimide (Sigma) was added to lyse the viable cells and a suspension of the cell nuclei was obtained and counted using a Neubauer chamber.

\section{Nitrites Quantification}

Nitrite production by macrophages was determined by the Griess assay (22).

\section{Cytokine Analysis by ELISA}

Tumor necrosis factor and IL-10 were measured in the culture supernatants using commercial kits (eBioscience) according to the manufacturer's specifications.

\section{Immunofluorescence}

Bone marrow-derived macrophages were cultured in cover-slips placed at the bottom of the 24 -well incubation plates, infected, and treated according to the description in the figure legends. At specific time points after infection, cells were fixed in $2 \%$ buffered paraformaldehyde (PFA) and probed with rabbit antihuman/mouse specific for active caspase 3 (R\&D Systems) or anti-mouse specific for p53 (Cell Signaling), followed by Alexa Fluor 594-conjugated goat anti-rabbit or anti-mouse IgG (Molecular Probes), respectively. 4',6-Diamino-2-phenylindole hydrochloride (DAPI) (Molecular Probes) was used to detect nuclei. Quantification of mean intensity of p53 protein was performed using the regions of interest (ROI) Manager application of the ImageJ program, which quantifies the mean intensity of selected areas. A total of five different fields for each cover-slip was analyzed. The mean intensity was calculated by selecting the ROI using the freehand selection tool. The number of caspase- 3 positive cells was determined by acquiring five different fields of the cover-slips and counting 300 cells for each condition. The percentage of caspase- 3 positive cells was calculated by dividing the number of caspase- 3 positive cells by the total number of cells.

\section{Quantitative Real-Time PCR Analysis}

Total RNA from cultured BMDM was extracted and reversetranscribed as described previously (20). Mouse p53, Bcl2, and Bax gene mRNA expression was quantified using specific primer probes (ABI) (Mm01731287_m1; Mm00477631_m1 and Mm00432051_m1, respectively) and normalized to the hypoxanthine guanine phosphoribosyl transferase (HPRT) mRNA levels (Mm00446968_m1).

\section{Cytochrome c Release}

The protocol was performed as previously described (23). Briefly, cells were permeabilized with digitonin buffer $(50 \mu \mathrm{g} /$ $\mathrm{mL}$ of digitonin in PBS with $100 \mathrm{mM} \mathrm{KCl})$, fixed in $2 \%(\mathrm{w} / \mathrm{v})$ buffered-PFA in PBS ( $\mathrm{pH} \mathrm{7.4),} \mathrm{for} 30 \mathrm{~min}$ at room temperature, rinsed, resuspended in $\mathrm{PBS}$, and stored at $4^{\circ} \mathrm{C}$. When all time points had been collected, $10^{6}$ cells were incubated in blocking buffer for $1 \mathrm{~h}$, at room temperature, stained with mouse purified anti-cytochrome $c$ antibody (BD Pharmingen) overnight at $4^{\circ} \mathrm{C}$, followed by the secondary anti-mouse IgG1-PE antibody (BioLegend) for $20 \mathrm{~min}$. Stained cells were run in a LSRII flow cytometer and analyzed with FlowJo software (Tristar). 


\section{Western Blot}

Protein extracts were prepared, resolved in 12\% SDS-PAGE, and western blot performed as described previously (24). Primary antibodies were actin (JLA20, developed by Lin JJC and obtained from the Developmental Studies Hybridoma Bank, developed under the auspices of the National Institute of Child Health
A

M. bovis BCG
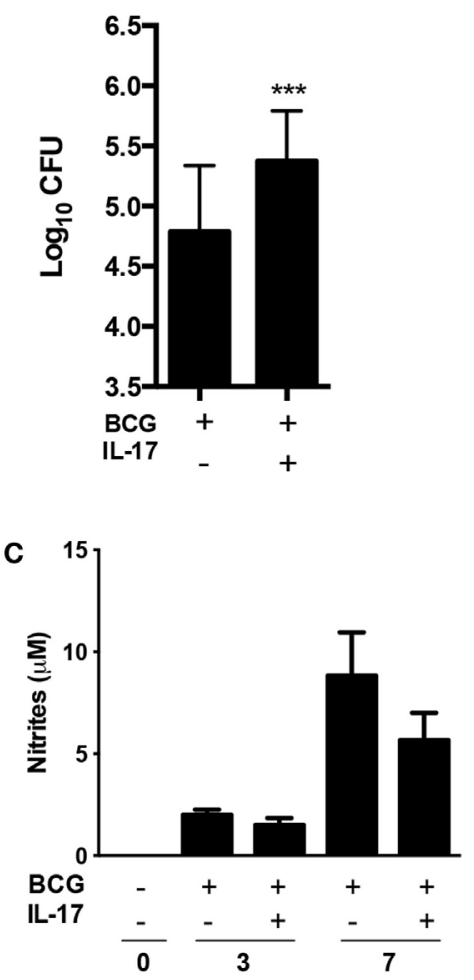

Time post-infection, days

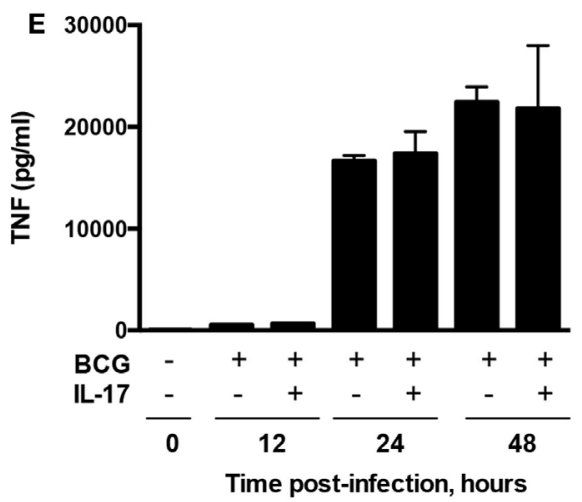

B M. tuberculosis

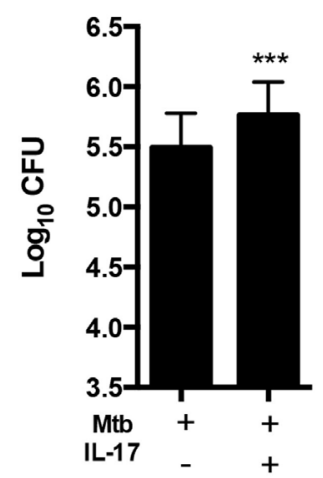

D
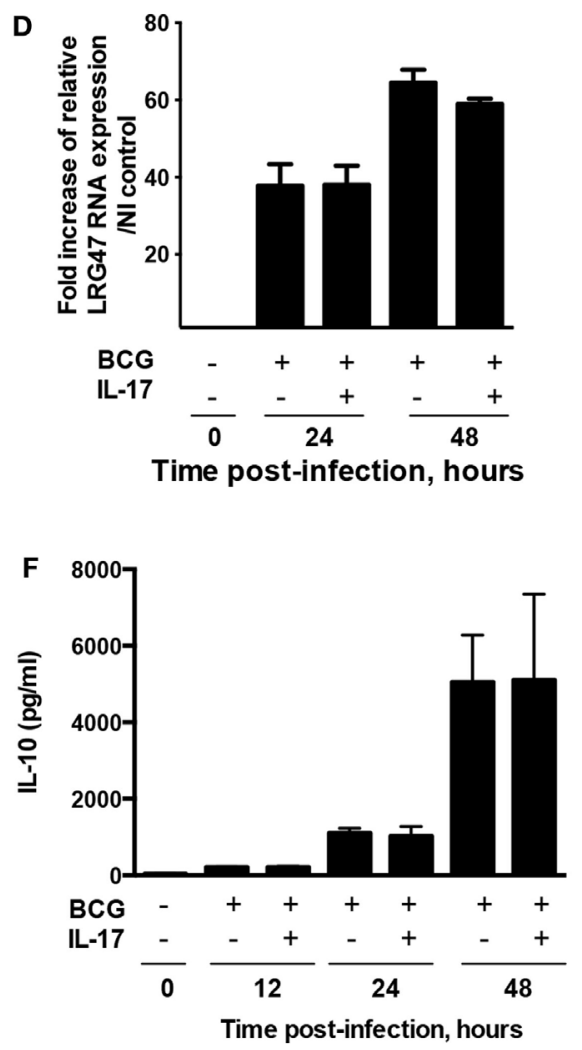

FIGURE 1 | IL-17 promotes growth of $\boldsymbol{M}$. bovis BCG and $\boldsymbol{M}$. tuberculosis in infected BMDM. BMDM were infected with $M$. bovis BCG (A) or with $M$. tuberculosis (B) and treated (+) or not (-) with IL-17. (A) Four days post-infection, the bacterial load was assessed. Fifty out of 50 independent experiments are represented in the graph. The mean-fold increase in CFUs induced by IL-17 is $0.4 \pm 0.2 \mathrm{log}$. (B) Three days post-infection, the bacterial load was assessed. Eleven out of 11 independent experiments are represented in the graph. The mean-fold increase in CFUs induced by IL-17 is $0.28 \pm 0.18 \mathrm{log}$. Significance determined by Student's $t$ test $\left.{ }^{\star \star \star *} p<0.001\right)$. BMDM were left uninfected or infected with M. bovis BCG and treated (+) or not $(-)$ with IL-17. (C) Nitrites production by was measured in the supernatants of the cultures by the Griess method. (D) The mRNA expression of LRG47 was quantified by real-time PCR using the primers (sense 5'-CTCTGGATCAGGGTTTGAGGAGTA-3'; anti-sense 5'-GGAACT GTGATGGTTTCATGATA-3') and probes (5'-LCred640-AGGTCCACAGACAGCGTCACTCGG -P-3'; 5'-AACCAGAGAGCCTCACCAGG GAGCTGA-FL-3') and normalized to HPRT. The fold increase of LRG47 mRNA expression over NI control was calculated. Represented are the mean \pm SE of three independent experiments. $(\mathbf{E}, \mathbf{F})$ At different time points post-infection, supernatants were harvested and the production of TNF (E) and IL-10 (F) assessed by immunoassay. Data point represents a mean of $n=6$. Results are from one representative out of three independent experiments. 

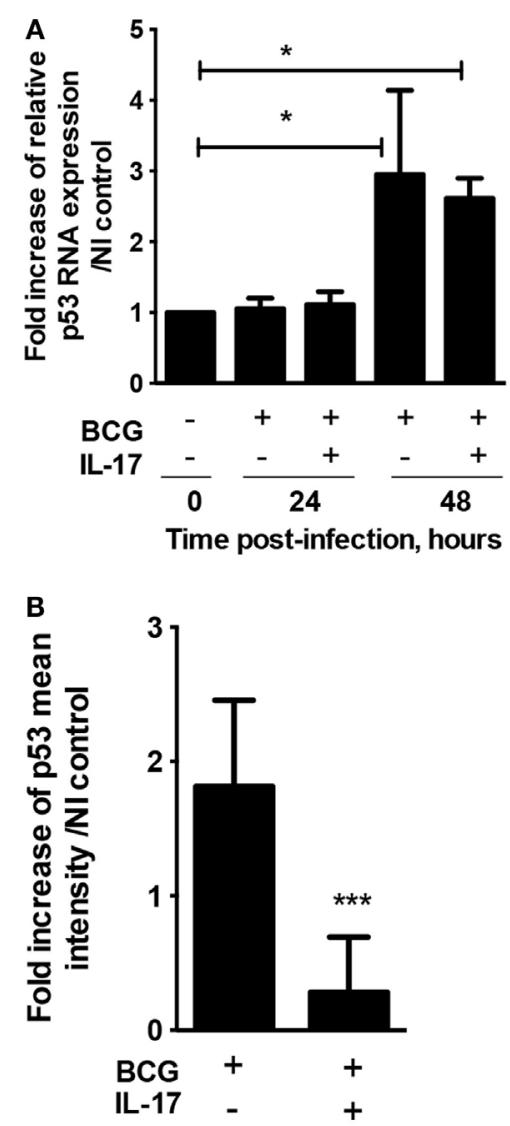

FIGURE 2 | IL-17 inhibits the upregulation of p53 observed during infection of BMDM with $\boldsymbol{M}$. bovis BCG. BMDM were infected with $M$. bovis BCG and treated (+) or not (-) with IL-17. (A) At the indicated time points, the expression of p53 was assessed by quantitative real-time PCR and normalized against HPRT. The fold increase of p53 mRNA expression over non-infected (NI) control was calculated. (B) Two days post-infection p53 levels were assessed by immunofluorescence and the fold increase of p53 mean intensity of cells over $\mathrm{NI}$ control calculated using ROI Manager application of the ImageJ program. The CFU controls for the represented experiments are plotted in Figure 1A. Representative images used for the calculations are in Figure S1 in Supplementary Material. Represented are the mean \pm SE of three independent experiments each of them performed for triplicate conditions. Significance determined by Student's $t$ test $\left({ }^{\star \star \star} p<0.001\right)$.

and Human Development and maintained by The University of Iowa, Department of Biology), Bax, Bcl2, and caspase-3 (all Cell Signaling). Secondary antibodies were from Southern Biotech or Santa Cruz. The same membrane was used to detect $\mathrm{Bax}$ and $\mathrm{Bcl} 2$. Total and cleaved caspase- 3 was detected in the same membrane. Chemiluminescence detection system SuperSignal West Femto (Thermo Scientific) and the Universal Hood II (Bio-Rad) were used as a detection system. Band intensity was quantified using QuantityOne (Bio-Rad).

\section{Statistical Analysis}

The results are given as mean \pm SE. Statistical significance was calculated by using Student's $t$ test, or one-way ANOVA with Tukey's post-test. Values of $p \leq 0.05$ were considered significant.

\section{Results}

\section{IL-17 Renders Macrophages More Permissive to} Mycobacterium Growth

IL-17 production is induced upon M. bovis BCG and M. tuberculosis infection, with $\gamma \delta \mathrm{T}$ cells as the major cellular source during the early stage of the infection $(19,25)$. However, the impact of this cytokine on mycobacterially infected macrophages remains to be investigated. To address this, mouse primary BMDM were infected with $M$. bovis BCG in the presence or absence of IL-17 and, 4 days after infection, the number of viable bacteria present in each condition was determined. A significantly higher number of viable bacteria were consistently found in macrophages stimulated with IL-17 (Figure 1A). Importantly, this effect was not specific for $M$. bovis BCG as the ability of macrophages to control M. tuberculosis was also significantly hampered by IL-17 (Figure 1B). These data suggest that IL-17 renders mycobacterially infected macrophages more permissive to bacterial growth. Notably, IL-17 did not interfere with basic macrophage microbicidal mechanisms, such as nitric oxide production (Figure 1C) or LRG47 expression, a marker for phagolysosome formation (Figure 1D). Since TNF and IL-10 play important roles on the regulation of apoptosis in mycobacterially infected macrophages $(11,12)$, a possible regulation of these molecules by IL-17 was next investigated. However, IL-17 did not impact the production of TNF (Figure 1E) or of IL-10 (Figure 1F) observed in macrophages upon $M$. bovis BCG infection.

\section{IL-17 Interferes with p53 in Mycobacterially Infected Macrophages}

Activation of the apoptotic pathway is thought to be a host defensive mechanism against mycobacterial pathogens (26). IL-17 has been shown to modulate apoptosis in viral infection (13) and in cancer $(17,18)$, but its role in the context of mycobacterial infection inducing apoptosis has not been addressed yet. Since IL-17 has been shown to down-regulate the activity of the apoptosis master regulator p53 $(27,28)$, we investigated whether IL-17 is able to modulate p53 in mycobacterially infected macrophages. While an upregulation of p53 transcription upon infection with $M$. bovis BCG was observed $48 \mathrm{~h}$ post-infection, this was not affected by the presence of IL-17 (Figure 2A). In contrast, the increase in $\mathrm{p} 53$ protein levels as measured by immunofluorescence, observed as early as 2 days post-M. bovis BCG infection, was abrogated by IL-17 (Figure 2B; Figure S1 in Supplementary Material). These data show that IL-17 down-regulates p53 at the protein level, suggesting that it may interfere with apoptosis of infected cells. Further, this interference may be the pathway by which IL-17 impacts the bacterial growth control.

\section{IL-17 Inhibits Apoptosis in Mycobacterially Infected Macrophages}

Given that IL-17 modulated p53 protein, this cytokine could impact on the induction of apoptotic programed cell death, an important antimicrobial mechanism operating upon mycobacterial infection (26). Thus, the role of IL-17 on the survival of infected macrophages was investigated. First, the viability of BMDM at different times post-infection with $M$. bovis BCG was measured. 

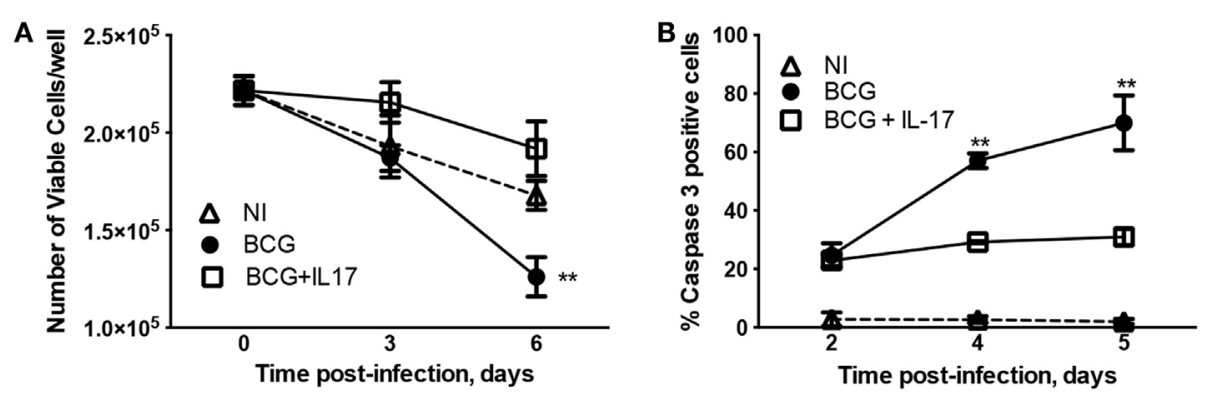

C

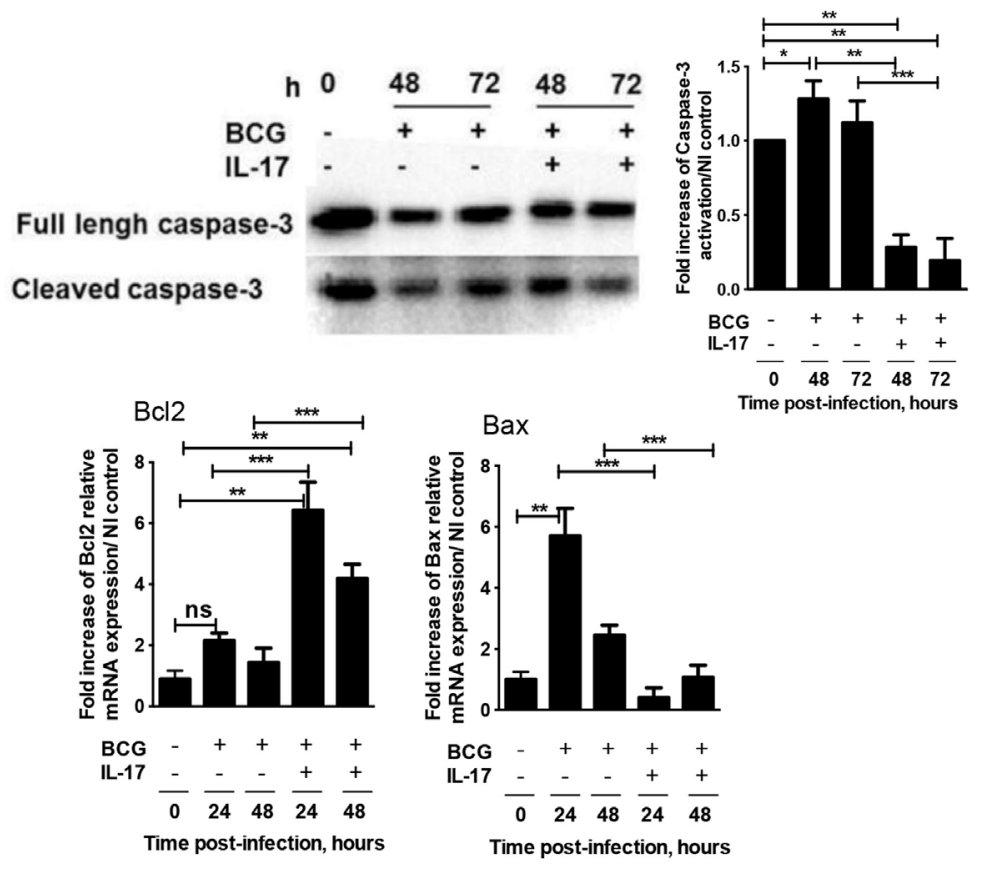

E
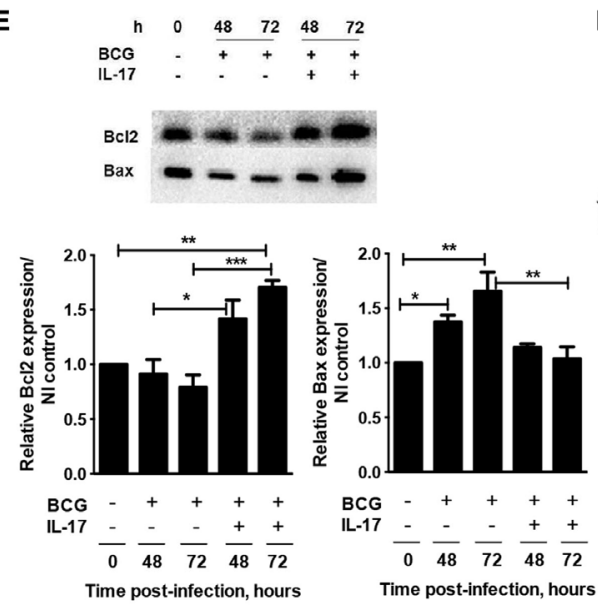

$\mathbf{F}$
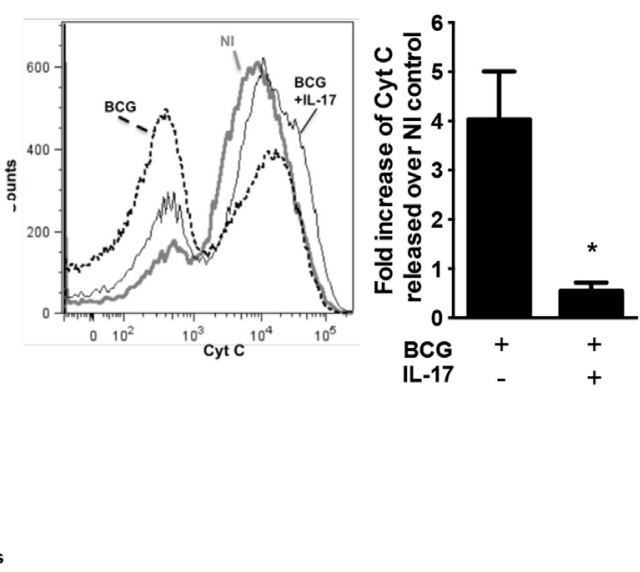

FIGURE 3 | IL-17 inhibits macrophage apoptosis induced by mycobacterial infection. BMDM were infected with $M$. bovis BCG in the presence or absence of IL-17 as indicated. (A) On days 2 and 7 post-infection, the cell viability was assessed by enumerating nuclei. (B) On days 2, 4, and 5 post-M. bovis BCG infection, activation of caspase-3 was assessed by immunofluorescence. (C) At 48 or $72 \mathrm{~h}$ post-M. bovis BCG infection, full-length and cleaved forms of caspase-3 protein were determined by western blot. (D,E) At 24 or $48 \mathrm{~h}$ post-M. bovis BCG infection, the Bcl2 and Bax mRNA or at 48 or $72 \mathrm{~h}$ post-M. bovis BCG infection, the protein were determined by real-time PCR or western blot respectively. (F) Cytochrome $c$ release from the mitochondria was determined 2 days post-infection by flow cytometry. The fold increase of cytochrome $c$ released over NI control was calculated for each independent experiment. Representative images used for (A) and (B) are in Figure S2 in Supplementary Material. Represented are the mean \pm SE of four independent experiments. Significance determined by one-way ANOVA (A,B) or Student's $t$ test (D-F) $\left({ }^{\star} p<0.05 ;{ }^{* *} p<0.01 ;{ }^{* \star *} p<0.001\right)$. 

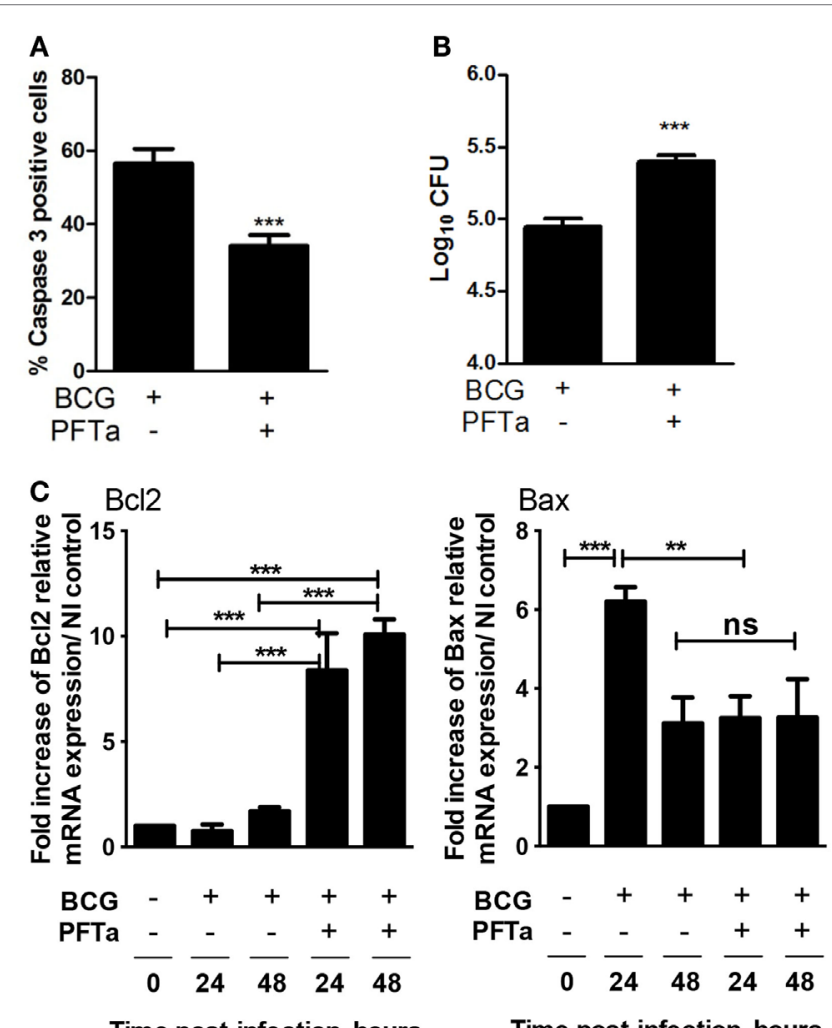

Time post-infection, hours

Time post-infection, hours

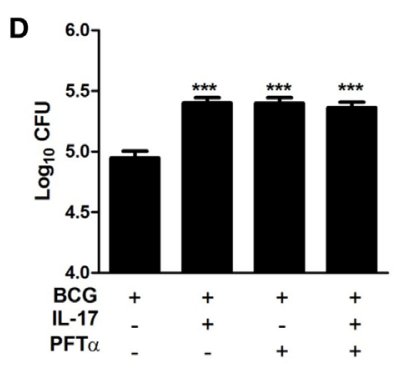

FIGURE 4 | Blockade of p53 impairs apoptosis of infected macrophages and bacterial growth control. BMDM were infected with $M$. bovis BCG in the presence (+) or absence (-) of the p53 inhibitor PFT $\alpha$. Four days post-infection (A) caspase-3 activation was assessed by immunofluorescence and (B) the bacterial load was assessed as indicated before. Representative images used for the calculations are in Figure S3 in Supplementary Material. (C) At 24 or $48 \mathrm{~h}$ post-M. bovis BCG infection, the $\mathrm{Bcl} 2$ and Bax mRNA was determined by real-time PCR. The fold increase of $\mathrm{Bcl} 2$ or Bax mRNA over the $\mathrm{NI}$ control was calculated for each independent experiment. (D) BMDM were left uninfected or infected with $M$. bovis BCG in the presence or absence of IL-17 or of PFT $\alpha$ as indicated. Four days post-infection the bacterial load was assessed as indicated before. Represented are the mean \pm SE of three independent experiments. Significance determined by Student's $t$ test $\left({ }^{\star \star \star} p<0.001\right)$.

As expected, the survival of infected macrophages decreased over time as compared to non-infected (NI) cells. However, the presence of IL-17 increased the survival of infected macrophages to levels similar to those of NI cells (Figure 3A; Figure S2A in Supplementary Material). Next, BMDM were infected with $M$. bovis BCG in the absence or presence of IL-17 and the activation of caspase-3, a major apoptotic effector caspase, was determined by immunofluorescence microscopy. We found that the presence of IL-17 resulted in a reduction in the percentage of caspase3-positive macrophages through day 5 of infection with $M$. bovis BCG (Figure 3B; Figure S2B in Supplementary Material) or with M. tuberculosis (Figure S2C in Supplementary Material). Additionally, and in line with the immunofluorescence data, we observed a decrease in the protein levels of activated (cleaved) caspase- 3 upon addition of IL-17 to BCG-infected macrophages, as determined by western blot (Figure 3C). Since our previous data (Figure 2) showed that IL-17 reduced p53 protein and since p53 is a master regulator of pro- and anti-apoptotic molecules (29), namely Bax and Bcl2, we next explored the impact of IL-17 on these molecules. Upon infection with M. bovis BCG, the transcription of $\mathrm{Bcl} 2$ was not much affected when compared to NI cells, whereas that of Bax was significantly increased (Figure 3D). In the presence of IL-17, a marked increase of $\mathrm{Bcl} 2$ transcription accompanied by a decrease of Bax mRNA was observed (Figure 3D). The increased $\mathrm{Bcl} 2$ transcription observed in the presence of IL-17 was confirmed at the protein level, as measured by western blot (Figure 3E). In contrast, addition of IL-17 did not affect the amount of Bax protein (Figure 3E). In all, in the presence of IL-17, the ratio of $\mathrm{Bcl} 2 / \mathrm{Bax}$ proteins was significantly increased, suggesting an anti-apoptotic activity for IL-17. The ratio of $\mathrm{Bcl} 2$ to $\mathrm{Bax}$ is an important component of the intrinsic apoptotic pathway that regulates the permeabilization of the outer mitochondrial membrane, and thereby the release of cytochrome $c$. Therefore, the release of cytochrome $c$ in $M$. bovis BCG-infected macrophages in the presence or absence of IL-17 was determined. We found that cytochrome $c$ release was observed upon infection of macrophages, in line with the activation of the intrinsic apoptotic pathway (Figure 3F). Further, it was clear that cytochrome $c$ release induced by BCG was abrogated in the presence of IL-17 (Figure 3F). These data suggest that IL-17 inhibits the intrinsic apoptotic pathway by altering the $\mathrm{Bcl} 2 / \mathrm{Bax}$ ratio and the release of cytochrome $c$.

\section{Modulation of p53 Activity Impacts Mycobacterial Growth in Macrophages}

As IL-17 decreases the capacity of macrophages to limit bacterial growth (Figure 1), reduces availability of p53, and limits apoptosis in mycobacterially infected macrophages, we hypothesized that IL-17 impacts mycobacterial growth via modulation of p53 activity. To test this hypothesis, we chemically inhibited the nuclear translocation of p53 during infection of macrophages with $M$. bovis BCG. Blocking the nuclear activity of p53 led to a significant decrease on the percentage of caspase 3-positive cells (Figure 4A and Figure S3A in Supplementary Material) accompanied by a significant increase in the number of bacteria (Figure 4B). Also, we observed that PFT- $\alpha$ addition to $M$. bovis BCG-infected macrophages increased the expression of the Bcl2, whereas that of Bax was not significantly altered (Figure 4C). The effect of PFT- $\alpha$ in increasing the number of bacteria was not further potentiated by IL-17 (Figure 4D), suggesting that p53 is a downstream mediator of IL-17 in this experimental setting. All our data show that IL-17, by modulating cell survival, impacts mycobacterial control by macrophages. 


\section{Discussion}

The importance of apoptosis in infection and of its modulation by intracellular pathogens is established. Apoptosis of mycobacterially infected cells is an important microbicidal mechanism, preventing the spread of the pathogen and promoting antigen cross-presentation (26). Whether the virulence of the infecting mycobacterial strain plays a role in promoting or evading apoptosis of the host cells is still a matter of debate $(3,4)$. However, it is clear that understanding the mechanisms underlying the fate of M. tuberculosis-infected macrophages is of critical importance, as therein may lay the potential to intervene. The programed cell death pathways in mycobacterially infected cells are regulated by host-encoded molecules, including eicosanoids $(5,6)$ and cytokines $(11,12)$. Our study adds a new layer to the complexity of this regulation, by reporting a pathway wherein IL-17 functions as an anti-apoptotic mediator in mycobacterially infected cells through its action on p53 and inhibition of the mitochondrial apoptotic pathway.

We dissected the early events of the apoptotic pathway initiated by mycobacterial infection. Following $M$. bovis BCG infection, p53 transcription occurred and there was an increase in the percentage of p53-positive cells, in agreement with previous studies (11). This was accompanied by a reduction in the $\mathrm{Bcl} 2 / \mathrm{Bax}$ ratio, the activation of caspase- 3 and an increase in cytochrome $c$ release. All these pro-apoptotic events were down-regulated by IL-17 activity, placing this cytokine as a novel apoptotic modulator in the context of mycobacterial infection. Several lines of evidence support a link between IL-17 and apoptosis, for example, in the modulation of Fas-Fas ligand induced apoptosis in the context of a viral infection (13), of fibroblast-like synoviocytes apoptosis, through the activation of STAT-3 (15), and in inhibition of $\mathrm{p} 53$ transcription in a lymphoma cell line (27). As we now show, in the context of mycobacteria infection, IL-17 acts by decreasing the levels of p53 protein in M. bovis BCG-infected BMDM. This modulation is likely taking place at the post-transcriptional level since alterations of p53 transcription were not observed in our experimental setting. An important control mechanism for p53 regulation is through ubiquitination, with several E3 ligases described for p53 (30). Act1, the key

\section{References}

1. WHO. Global Tuberculosis Report 2014. Geneva: World Health Organization (2014).

2. O'Garra A, Redford PS, McNab FW, Bloom CI, Wilkinson RJ, Berry MP. The immune response in tuberculosis. Annu Rev Immunol (2013) 31:475-527. doi:10.1146/annurev-immunol-032712-095939

3. Aguilo N, Marinova D, Martin C, Pardo J. ESX-1-induced apoptosis during mycobacterial infection: to be or not to be, that is the question. Front Cell Infect Microbiol (2013) 3:88. doi:10.3389/fcimb.2013.00088

4. Parandhaman DK, Narayanan S. Cell death paradigms in the pathogenesis of Mycobacterium tuberculosis infection. Front Cell Infect Microbiol (2014) 4:31. doi:10.3389/fcimb.2014.00031

5. Chen M, Gan H, Remold HG. A mechanism of virulence: virulent Mycobacterium tuberculosis strain H37Rv, but not attenuated H37Ra, causes significant mitochondrial inner membrane disruption in macrophages leading to necrosis. J Immunol (2006) 176:3707-16. doi:10.4049/jimmunol. 176.6.3707 transducer of IL-17 receptor signaling, is also an E3 ubiquitin ligase, which shares conserved residues with those known to ubiquitinate p53 and activate its proteasomal degradation (31). It is, therefore, tempting to speculate that IL-17 may be promoting the degradation of $\mathrm{p} 53$ through proteasome degradation. It will be important in future to further dissect the IL-17-driven upstream events leading to the observed alterations, particularly those happening immediately after IL-17R triggering. Mechanistically, it is possible that the effect of IL-17 in blocking apoptosis of the infected cell requires other molecules produced during infection. Although the balance between TNF and IL-10 within mycobacterially infected macrophages is important in the occurrence of apoptosis of the infected cell (12), we did not observe an impact of IL-17 in the amount of secreted TNF or IL-10 produced.

Elucidating the mechanisms by which IL-17-mediates p 53 modulation is central not only in the context of intracellular infections, but also considering the fact that several studies involving the axis IL-17/p53 in cancer have been undertaken (27, 28,32 ) and that IL-17-based therapies are being developed (33).

\section{Acknowledgments}

The authors thank the personnel at the ICVS animal house facility for excellent animal husbandry. This work has been funded by Fundação para a Ciência e Tecnologia, Portugal. Project grants: PTDC/SAU-MII/101977/2008 (to AGC), PTDC/ BIA-BCM/102776/2008 (to MS) and HMSP-ICT/0024/2010 (to RA) and co-funded by Programa Operacional Regional do Norte (ON.2 - O Novo Norte), Quadro de Referência Estratégico Nacional (QREN), through the Fundo Europeu de Desenvolvimento Regional (FEDER). Personal Grants: SFRH/ BPD/33036/2006 to AC; SFRH/BD/33573/2009 to JBG. AMC is supported by Trudeau Institute, Inc. and NIAID PO1 AI46530. MS is a FCT Associate Investigator.

\section{Supplementary Material}

The Supplementary Material for this article can be found online at http://journal.frontiersin.org/article/10.3389/fimmu.2015.00498

6. Divangahi M, Chen M, Gan H, Desjardins D, Hickman TT, Lee DM, et al. Mycobacterium tuberculosis evades macrophage defenses by inhibiting plasma membrane repair. Nat Immunol (2009) 10:899-906. doi:10.1038/ni.1758

7. Schaible UE, Winau F, Sieling PA, Fischer K, Collins HL, Hagens K, et al. Apoptosis facilitates antigen presentation to $\mathrm{T}$ lymphocytes through MHC-I and CD1 in tuberculosis. Nat Med (2003) 9:1039-46 doi:10.1038/nm906

8. Sly LM, Hingley-Wilson SM, Reiner NE, McMaster WR. Survival of Mycobacterium tuberculosis in host macrophages involves resistance to apoptosis dependent upon induction of antiapoptotic Bcl-2 family member Mcl-1. J Immunol (2003) 170:430-7. doi:10.4049/jimmunol.170.1.430

9. Aporta A, Arbues A, Aguilo JI, Monzon M, Badiola JJ, de Martino A, et al. Attenuated Mycobacterium tuberculosis SO2 vaccine candidate is unable to induce cell death. PLoS One (2012) 7:e45213. doi:10.1371/journal. pone.0045213

10. Seimon TA, Kim MJ, Blumenthal A, Koo J, Ehrt S, Wainwright H, et al. Induction of ER stress in macrophages of tuberculosis granulomas. PLoS One (2010) 5:e12772. doi:10.1371/journal.pone.0012772 
11. Rodrigues MF, Barsante MM, Alves CC, Souza MA, Ferreira AP, AmaranteMendes GP, et al. Apoptosis of macrophages during pulmonary Mycobacterium bovis infection: correlation with intracellular bacillary load and cytokine levels. Immunology (2009) 128:e691-9. doi:10.1111/j.1365-2567.2009.03062.x

12. Rojas M, Olivier M, Gros P, Barrera LF, Garcia LF. TNF-alpha and IL-10 modulate the induction of apoptosis by virulent Mycobacterium tuberculosis in murine macrophages. J Immunol (1999) 162:6122-31. doi:10.1084/ jem. 20082030

13. Hou W, Kang HS, Kim BS. Th17 cells enhance viral persistence and inhibit T cell cytotoxicity in a model of chronic virus infection. J Exp Med (2009) 206:313-28. doi:10.1084/jem.20082030

14. Sergejeva S, Ivanov S, Lotvall J, Linden A. Interleukin-17 as a recruitment and survival factor for airway macrophages in allergic airway inflammation. Am J Respir Cell Mol Biol (2005) 33:248-53. doi:10.1165/rcmb.2004-0213OC

15. Lee SY, Kwok SK, Son HJ, Ryu JG, Kim EK, Oh HJ, et al. IL-17-mediated Bcl-2 expression regulates survival of fibroblast-like synoviocytes in rheumatoid arthritis through STAT3 activation. Arthritis Res Ther (2013) 15:R31. doi:10.1186/ar4179

16. Song X, Qian Y. IL-17 family cytokines mediated signaling in the pathogenesis of inflammatory diseases. Cell Signal (2013) 25:2335-47. doi:10.1016/j. cellsig.2013.07.021

17. Wilke CM, Kryczek I, Wei S, Zhao E, Wu K, Wang G, et al. Th17 cells in cancer: help or hindrance? Carcinogenesis (2011) 32:643-9. doi:10.1093/ carcin/bgr019

18. Zou W, Restifo NP. T(H) 17 cells in tumour immunity and immunotherapy. Nat Rev Immunol (2010) 10:248-56. doi:10.1038/nri2742

19. Lockhart E, Green AM, Flynn JL. IL-17 production is dominated by gammadelta T cells rather than CD4 T cells during Mycobacterium tuberculosis infection. J Immunol (2006) 177:4662-9. doi:10.4049/jimmunol.177.7.4662

20. Carmona J, Cruz A, Moreira-Teixeira L, Sousa C, Sousa J, Osorio NS, et al. Mycobacterium tuberculosis strains are differentially recognized by TLRs with an impact on the immune response. PLoS One (2013) 8:e67277. doi:10.1371/ journal.pone.0067277

21. Oliveira MS, Fraga AG, Torrado E, Castro AG, Pereira JP, Filho AL, et al. Infection with Mycobacterium ulcerans induces persistent inflammatory responses in mice. Infect Immun (2005) 73:6299-310. doi:10.1128/ IAI.73.10.6299-6310.2005

22. Stuehr DJ, Gross SS, Sakuma I, Levi R, Nathan CF. Activated murine macrophages secrete a metabolite of arginine with the bioactivity of endothelium-derived relaxing factor and the chemical reactivity of nitric oxide. $J \operatorname{Exp}$ Med (1989) 169:1011-20. doi:10.1084/jem.169.3.1011

23. Waterhouse NJ, Trapani JA. A new quantitative assay for cytochrome c release in apoptotic cells. Cell Death Differ (2003) 10:853-5. doi:10.1038/ sj.cdd. 4401263

24. Gama JB, Ohlmeier S, Martins TG, Fraga AG, Sampaio-Marques B, Carvalho MA, et al. Proteomic analysis of the action of the Mycobacterium ulcerans toxin mycolactone: targeting host cells cytoskeleton and collagen. PLoS Negl Trop Dis (2014) 8:e3066. doi:10.1371/journal.pntd.0003066

25. Umemura M, Yahagi A, Hamada S, Begum MD, Watanabe H, Kawakami K, et al. IL-17-mediated regulation of innate and acquired immune response against pulmonary Mycobacterium bovis bacille Calmette-Guerin infection. J Immunol (2007) 178:3786-96. doi:10.4049/jimmunol.178.6.3786

26. Behar SM, Martin CJ, Booty MG, Nishimura T, Zhao X, Gan HX, et al. Apoptosis is an innate defense function of macrophages against Mycobacterium tuberculosis. Mucosal Immunol (2011) 4:279-87. doi:10.1038/mi.2011.3

27. Li Q, Xu X, Zhong W, Du Q, Yu B, Xiong H. IL-17 induces radiation resistance of B lymphoma cells by suppressing p53 expression and thereby inhibiting irradiation-triggered apoptosis. Cell Mol Immunol (2015) 12:366-72. doi: $10.1038 / \mathrm{cmi} .2014 .122$

28. Radosavljevic G, Ljujic B, Jovanovic I, Srzentic Z, Pavlovic S, Zdravkovic $\mathrm{N}$, et al. Interleukin-17 may be a valuable serum tumor marker in patients with colorectal carcinoma. Neoplasma (2010) 57:135-44. doi:10.4149/ neo_2010_02_135

29. Nikulenkov F, Spinnler C, Li H, Tonelli C, Shi Y, Turunen M, et al. Insights into p53 transcriptional function via genome-wide chromatin occupancy and gene expression analysis. Cell Death Differ (2012) 19:1992-2002. doi:10.1038/ cdd.2012.89

30. Hock AK, Vousden KH. The role of ubiquitin modification in the regulation of p53. Biochim Biophys Acta (2014) 1843:137-49. doi:10.1016/j. bbamcr.2013.05.022

31. Liu C, Qian W, Qian Y, Giltiay NV, Lu Y, Misra S, et al. Act1, a novel U-box E3 ubiquitin ligase for IL-17R-mediated signaling. Sci Signal (2009) 2:ra63. doi:10.1126/scisignal.2000382

32. Xu B, Guenther JF, Pociask DA, Wang Y, Kolls JK, You Z, et al. Promotion of lung tumor growth by interleukin-17. Am J Physiol Lung Cell Mol Physiol (2014) 307:L497-508. doi:10.1152/ajplung.00125.2014

33. van den Berg WB, McInnes IB. Th17 cells and IL-17 a - focus on immunopathogenesis and immunotherapeutics. Semin Arthritis Rheum (2013) 43:158-170. doi:10.1016/j.semarthrit.2013.04.006

Conflict of Interest Statement: The authors declare that the research was conducted in the absence of any commercial or financial relationships that could be construed as a potential conflict of interest.

Copyright (C) 2015 Cruz, Ludovico, Torrado, Gama, Sousa, Gaifem, Appelberg, Rodrigues, Cooper, Pedrosa, Saraiva and Castro. This is an open-access article distributed under the terms of the Creative Commons Attribution License (CC BY). The use, distribution or reproduction in other forums is permitted, provided the original author(s) or licensor are credited and that the original publication in this journal is cited, in accordance with accepted academic practice. No use, distribution or reproduction is permitted which does not comply with these terms. 